\title{
Unmuting Ube3a in mice alleviates Angelman syndrome
}

Harnessing the silenced, but functional, paternal copy of the UBE3A gene via antisense oligonucleotides (ASOs) might provide a way to alleviate symptoms of Angelman syndrome. According to new research published in Nature, unsilencing paternal Ube3a in a mouse model of

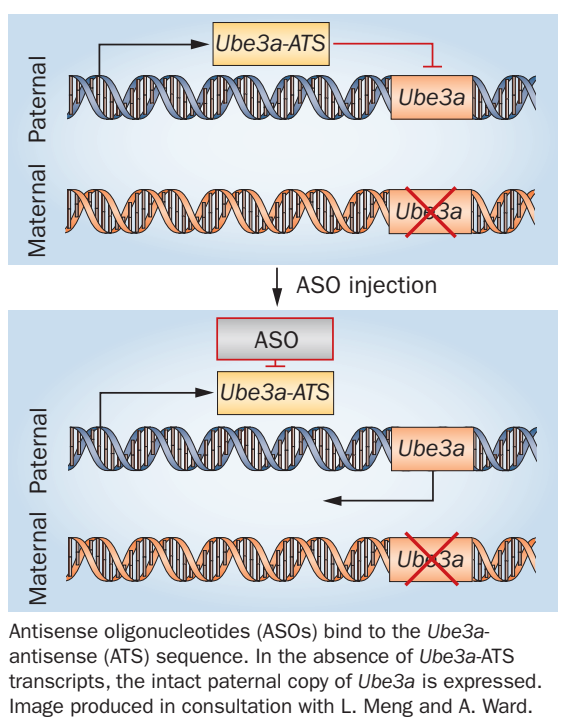

Angelman syndrome resulted in partial restoration of UBE3A protein expression and the reversal of memory deficits.

Angelman syndrome is a developmental disorder characterized by intellectual disability, including severely impaired speech. Often, individuals with the disorder also have ataxia, seizures and sleep disturbances.

Angelman syndrome is caused by a mutation or deletion of the maternal copy of $U B E 3 A$, leaving individuals with the syndrome with no functional copy of the gene in the CNS because the paternal copy of $U B E 3 A$ is silenced in neurons.

Arthur Beaudet's group from the Baylor College of Medicine, TX, USA, had previously discovered that silencing of the paternal $U B E 3 A$ was mediated by an antisense transcript (ATS). Stopping the expression of Ube3a-ATS might, therefore, reactivate the paternal copy of the gene.

Beaudet and colleagues at Isis Pharmaceuticals designed ASOs complementary to Ube3a-ATS, and injected them into the CNS of Angelman syndrome model mice. A single administration of the Ube3a-ATS ASOs was capable of unsilencing paternal Ube $3 a$ for 4 months, and abolished memory deficits previously seen on a contextual freezing task.

Ube3a-ATS ASO injection did not affect the motor deficits observed in the Angelman syndrome mice. Complete phenotypic reversal of the symptoms might require treatment before a critical developmental window has closed.

However, the researchers are cautiously optimistic. "It is my hope, and the hope of families who are dealing with Angelman syndrome, that these findings will support the advancement of a new therapy into clinical studies for the treatment of this disease," says Beaudet.

\section{Hemi Malkki}

Original article Meng, L. et al. Towards a therapy for Angelman syndrome by targeting a long non-coding RNA. Nature doi:10.1038/nature13975 